\title{
Correction to: Proceedings of the Thirteenth International Conference on Management Science and Engineering Management
}

Jiuping Xu, Syed Ejaz Ahmed, Fang Lee Cooke, and Gheorghe Duca

\section{Correction to:}

J. Xu et al. (Eds.): Proceedings of the Thirteenth

International Conference on Management Science

and Engineering Management, AISC 1002, https://doi.org/10.1007/978-3-030-21255-1

The original version of the book was inadvertently published with the following incorrect author affiliations:

For authors "Shuhua Hou" and "Rui Qiu", the affiliation "School for Environment and Sustainability, University of Michigan, Ann Arbor, USA" has been added and removed, respectively, in the chapter "A Multiple Decision-Maker Model for Construction Material Supply Problem Based on Costs-Carbon Equilibrium.”

Institute and department details have been added to the affiliation "Business School, Sichuan University, Chengdu, People's Republic of China" in the chapter "Customer Relationship and Efficiency Analysis of the Listed Air Companies in China."

The erratum chapters and book have been updated with the changes.

The updated versions of these chapters can be found at https://doi.org/10.1007/978-3-030-21255-1_37

https://doi.org/10.1007/978-3-030-21255-1_43

https://doi.org/10.1007/978-3-030-21255-1 